of other planets, and if there had ever been any difference, which is unlikely, considering the general unity of the solar system, it must long ago have disappeared in consequence of this interchange.

The argument is strengthened by what we know of the atmospheres of the planets, especially of our nearest neighbors, Mars and Venus. Not only do these planets give plain indications of their atmospheres, but it is certain that they are very much like our own. That is found out in the following way: Amongst the many dark lines, Fraunhofer lines, as they are called, in the solar spectrum there are certain well marked groups which Sir D. Brewster long ago pointed out to be due to the absorption of rays by our atmosphere, because they are seen to be blacker and more intense when the sun is low than when he is high in the sky. That is because the rays have to pass through a greater thickness of air before they reach us when the sun is nearer the horizon. Now, by carefully observing the light reflected from Venus and Mars, which must have twice passed through so much of their atmospheres as lies above the reflecting surface, it has been found that precisely the same rays which are darker when the sun is low are also darker in the spectra of these planets. Moreover, in these planets there are no new dark lines indicating any absorbent of a different kind. The more distant planets show additional absorption bands, but their atmospheres must, on account of their greater masses, perhaps also from lower temperature, be denser, and besides they appear to be full of clouds which may not be merely water-dust, and may well produce their own absorptive effects.

The argument, however, reaches a good deal farther. Not only are the planets moving through the so-called planetary space, but the sun and all its train are moving through the interstellar space. Astronomers are agreed that we are moving, but the direction of the movement is much better known than the pace. The rate is sometimes set down at about thirty miles a second: certainly not an extravagant estimate. But at any rate we are going, and leaving the interplanetary atmosphere, or some of it, behind. Even if the solar system had no such motion, the process of diffusion must gradually carry the interplanetary atmosphere into regions beyond, and, unless this diffusion were compensated by accession of air from without, the planets must gradually lose their atmospheres until the loss was stopped by the cooling effect before mentioned. After countless ages we have manifestly not reached that stage, so we must conclude that interstellar space is pervaded by an atmosphere, though it be of very great tenuity.

If this atmosphere is not of similar chemical constitution to our own, ours must be changing by slow degrees, and in course of ages the change must tell. There is, however, no reason to think that our atmosphere has for millions of years undergone any change sufficient to affect the constitution of animal life of the higher types, and if that be so the air of stellar space must be much the same as that of interplanetary space and our own. Sterry Hunt, from the preponderance of vegetable growth at certain periods of the earth's history, inferred that at those periods there must have been an excessive quantity of carbonic acid in the atmosphere; and he fancied that this was acquired from the stellar space as the solar system made its way into regions where there was an unusual amount of carbonic acid. Spectrum analysis has not led us to think that the chemical elements of the stars of any region are different from those with which we are acquainted in the earth and in the sun. Stars in the same region are mostly of the same type, and the types are few, and all the common types of spectra of stars give indications of elements which we know, and no certainty of any other elements. Distance makes no difference at all. The few stars with unusual spectra do not so much seem to have peculiar elements as to be in peculiar physical states. The ùniverse seems, so far, of one make, and there are no facts which negative the supposition that the whole vast space through which we see stars is filled with air; air very rare indeed, perhaps not a millionth of a millionth as dense as ours, but still, on the whole, similarly constituted.

\section{FISH ACCLIMATIZATION ON THE PACIFIC COAST.}

BY HUGH M. SMITH, M. D., UNITED STATES FISH COMMISSION.

Few experiments in fish culture have been economically more important and successful than those which have been .conducted by the United States Fish Commission with reference to the Pacific Coast. Coincident with the propagation of native fishes the introduction of non-indigenous species has been undertaken, with results that have been extremely gratifying to fish culturists, and perhaps more striking than any previously obtained in this or any other country.

Among the fishes inhabiting the rivers and coast waters of the Atlantic slope, none is better known, more important, and more highly esteemed than the shad (Clupea sapidissima) and the striped bass or rockfish (Roccus lineatus), the former being a food fish, pure and simple, the latter combining a gamey disposition with excellent food qualities. These fish are anadromous, entering the fresh water for the purpose of spawning and passing a large part of the year at sea or in the salt water. Attention. will be called to the experimental introduction of these fishes to the west coast, although several other important food-fish, among them the black bass (Micropterus salmoides) and catfish (Ameiurus nebulosus) might also be mentioned in this connection.

The introduction of shad fry to the west coast was first undertaken as long ago as 1871, when 12,000 young fish were deposited in the Sacramento River, under the auspices of the California Fish Commission. After that the experiment was taken up by the U. S. Fish Commission and carried on until 1886, during which time 609,000 young shad were placed in the Sacramento River, 600,000 in the Willamette River, 300,000 in the Columbia River and 10,000 in the Snake River.

Two or three years after the first fish were planted a few more or less mature examples were obtained in the Sacramento River; as additional deposits were made, the number of marketable fish began to increase, and the fish gradually distributed themselves along the entire coast of the United States north of Monterey Bay, until finally they have come to rank next to salmon in abundance among the river fishes of the west coast.

The U. S. Commissioner of Fish and Fisheries, in his annual report for 1887, speaking of the small plants of shad fry made in the Sacramento River at Tehama, says:

"From these slender colonies, aggregating less than one per cent of the number now annually planted in our Atlantic slope rivers, the shad have multiplied and distributed themselves along 2,000 miles of coast from the Golden Gate of California to Vancouver Island in British Columbia. They are abundant in some of the rivers, common in most of them, and occasional ones may be found everywhere in the estuaries and bays of this long' coast line.

"Prior to our experiments on the west coast it was a dictum of fish culture that fish planted in a river would return to it when mature for the purpose of spawning. The result of these experiments has been to demonstrate 
that this instinct of nativity, should it really exist, is in this case dominated by other influences, which have dispersed the shad planted in the Sacramento widely beyond the limits which we had assigned to them, and in the most unexpected direction.

"The cause is probably to be sought in the genial influences of the Japan current, which brings the warmth of equatorial Asia to temper the extremes of Arctic climate on the southern shore of the Alaskan Peninsula, and thence sweeping to the south, carries tropical heats to the latitude of San Francisco. Repelled on the one hand by the low temperature of the great rivers and fringe of coast waters, and solicited on the other by the equable and higher temperature of the Japan current, the shad have become true nomads, and have broken the bounds of the hydrographic area to which we had supposed they would be restricted. Following the track of the Asiatic current, and finding more congenial temperatures as they progress, it is not unreasonable to expect that some colonies will eventually reach the coast of Asia and establish themselves in its great rivers."

Shad are now found in greatest numbers in the Sacramento and Columbia Rivers, where they are of considerable economic value. Owing to the fact that very little apparatus specially adapted to their capture is employed, no correct idea of their actual abundance in a given stream can be formed. Nearly all the shad thus far taken have been obtained in nets operated for salmon or other fish, shad being only an incidental element in the catch. The price received by the fishermen is a good criterion of the abundance of the fish. When first taken, shad brought as much as $\$ 1.20$ a pound; in 1892 the value in many places was only two cents a pound, and in the Columbia River at one period the catch was so large and the price so low that the fishermen did not go to the trouble of marketing the fish caught. The average price on the coast has declined in the past four years from ten cents per pound in 1889 to four cents in 1892 .

An inquiry conducted by the U. S. Fish Commission in 1892 placed that bureau in possession of information showing the extent of the shad fishery in every river of the Pacific States. It was ascertained that in the year named 660,000 pounds of shad were marketed, the value of the same to the fishermen being about $\$ 27,000$. Reports received during the present year indicate a catch of perhaps a million pounds, and it seems reasonable to anticipate a steady increase in the production with the improved facilities for shipment and the growing demand for fresh fish in the rising towns adjacent to the coast rivers. A careful estimate places the total value of the shad catch on the Pacific coast to date at $\$ 145,000$, representing over $3,000,000$ pounds, while the aggregate outlay for all purposes connected with the introduction of the fry was less than $\$ 4,000$. This is certainly a satisfactory investment of the people's money.

The absence of a special scientific inquiry precludes the possibility of chronicling the changes which have probably been wrought in the habits of the shad as a result of the changed physical surroundings, thermic conditions, enemies and food supply. It may be noted, however, that the characteristic habit on the east coast of periodically ascending the rivers for the purpose of spawning and of returning, after the completion of that process, to the open sea where the principal part of the life of the fish is spent, appears to be considerably modified, in California, at least, where, in certain bays and estuaries, the shad is found in greater or less abundance during every month in the year. The evidence at hand indicates a condition prevailing in the littoral and fluvial waters of the Pacific coast that is very favorable to the growth of the shad. It is not unusual to take examples consider- ably larger than any ever seen in the eastern rivers. The average weight of the shad caught on the Atlantic coast is under four pounds, and the capture of fish weighing seven, eight or nine pounds is extremely rare. In California, however, it is not uncommon to secure shad weighing eight or ten pounds and reports have been made that fifteen-pound individuals have occasionally been obtained in salmon nets.

Of scarcely less consequence than the actual results of shad introduction on the west coast is the important bearing which the success of the experiment must have in determining the outcome of artificial propagation in regions in which it is not possible to distinguish with satisfactory accuracy the natural from the artificial conditions. If these far-reaching, and no doubt permanent, results attend the planting, on few occasions, of small numbers of fry in waters to which the fish are not indigenous, is it not permissible to assume that much more striking consequences must follow the planting of enormous quantities of fry, year after year, in native waters? There is no reasonable doubt that the perpetuation of the extensive shad fisheries in most of the rivers of the Atlantic coast has been accomplished entirely by artificial propagation. On no other supposition can the maintenance and increase of the supply be accounted for.

The introduction of the striped bass was accomplished in 1879, when about 150 fish a few inches long, taken in Shrewsbury River, N. J., were successfully carried across the continent and deposited at the mouth of the Sacramento River by an agent of the U.S. Fish Commission co-operating with the California Commission. Six or seven months later an example eight inches in length was reported from Monterey Bay, one hundred miles south of the locality where planted, and in eleven months another specimen twelve and a half inches long, and weighing one pound, was caught in San Francisco harbor. This very rapid growth indicated the special adaptability of the waters of the region to this fish. In 1882 another plant, consisting of 300 fish, was made in the same region by the California authorities. As a result of these two small deposits, the species soon became distributed along the entire coast of California; its occurrence, however, in the other States of the region has not yet been determined.

The history of the striped bass is similar to that of the shad. It has attained considerable commercial importance, has increased steadily and rapidly, and is generally regarded as one of the best food fishes of the coast. It has not yet attained anything like the abundance of the shad, nor was this to have been expected from the meagre plants, but there seems to be no reason to doubt that it is only a question of time when it will become one of the most prominent economic fishery products of the region as well as a favorite object of capture by sportsmen.

The largest quantities of striped bass are taken for market in San Francisco Bay with seines and gill nets. The fish are found in greatest numbers between October 1 and February 15, but occur in some abundance at all seasons. Their average weight is eight or ten pounds, but fish weighing forty pounds are not scarce. The estimation in which they are held may be judged from the market value. In 1888, the ruling price in San Francisco was one dollar a pound; in 1892, owing to an increased pro'duction, it had dropped to twelve and a half cents. 'The catch in the latter year was about 43,000 pounds, for which the fishermen received $\$ 5,350$. The aggregate yield to date may be estimated at nearly 100,000 pounds, with a value at first hands of about $\$ 18,000$. The transportation of striped bass to the Pacific being undertaken conjointly with that of a number of other fishes, it is probable that the proportional cost of introduction was not more than a few hundred dollars. 